\title{
Differences between radial and ulnar deviation of the wrist in the study of the intrinsic intercarpal ligaments: magnetic resonance imaging and gross anatomic inspection in cadavers
}

\author{
Ramon Gheno • Florian M. Buck • Marcelo A. C. Nico • \\ Debra J. Trudell • Donald Resnick \\ Received: 9 June 2009/Revised: 4 August 2009 / Accepted: 20 August 2009/Published online: 10 October 2009 \\ (C) The Author(s) 2009. This article is published with open access at Springerlink.com
}

\begin{abstract}
Objective To demonstrate how radial and ulnar deviation of the wrist can affect the visualization of the intrinsic intercarpal ligaments using magnetic resonance (MR) imaging, MR arthrography and gross anatomic inspection in cadavers.

Materials and methods The detectability of the intrinsic intercarpal ligaments of ten fresh human wrists was analyzed in coronal, axial and sagittal images in the neutral position and in radial and ulnar deviation with MR imaging and MR arthrography. The findings were then correlated with gross anatomic inspection. Additionally, quantitative measurements including the radiocarpal distances and capitate angles were performed.

Results Differences were noted in the visual conspicuity of only the intercarpal ligaments of the proximal carpal row with different techniques and wrist positions. The average width of the radiocarpal joint was $0.62 \mathrm{~mm}, 1.55 \mathrm{~mm}$ and $2.0 \mathrm{~mm}$ (radial side) and $3.78 \mathrm{~mm}, 2.25 \mathrm{~mm}$ and $1.16 \mathrm{~mm}$ (ulnar side) in radial deviation, neutral position, and ulnar deviation of the wrist, respectively. Statistically, these maneuvers produced significant opening in the ulnar side during radial deviation (Student's $t$-test; $P=0.0005)$ and in the radial side in ulnar deviation $(P=0.007)$.

Conclusion Significant differences in the width of the radiocarpal joint were observed during radial and ulnar
\end{abstract}

R. Gheno · F. M. Buck • M. A. C. Nico • D. J. Trudell • D. Resnick Department of Radiology, VA San Diego Medical Center,

San Diego, CA, USA

R. Gheno $(\bowtie)$

Rua Gonçalves Ledo 776/303,

Porto Alegre, RS 90610250, Brazil

e-mail: rgheno@yahoo.com deviation of the wrist, influencing the visualization of the intrinsic ligaments, mainly the scapholunate and lunotriquetral ligaments. The use of MR arthrography with radial and/or ulnar deviation has the potential to improve diagnosis in clinical cases in which injury to one or both of these ligaments is suggested.

Keywords Wrist · MRI · Ligaments · Arthography

\section{Introduction}

The ligaments of the wrist play a major role in guiding and restraining motion of the carpal bones. Although some injuries of the carpal ligaments may have little clinical importance, many are associated with pain and instability and can benefit from surgical intervention [1-7].

Despite some controversies in the classification of the wrist ligaments, they can generally be divided into intrinsic and extrinsic categories, as described by Taleisnik [7]. Intrinsic ligaments are entirely within the carpus (attaching only to carpal bones) [7-10]. The most important intrinsic carpal ligaments are the scapholunate (SL), lunotriquetral (LT), trapeziotrapezoid (TT), capitotrapezoid (CT), capitohamate $(\mathrm{CH})$, triquetrocapitate $(\mathrm{TC})$, triquetrohamate $(\mathrm{TH})$, scaphotrapeziotrapezoid (STT), and scaphocapitate (SC) ligaments (Figs. 1 and 2). The ligaments attaching to the pisiform bone are generally not considered as intrinsic, owing to the fact that this bone is believed to be sesamoid in nature [1]. Extrinsic ligaments connect the bones of the forearm (i.e., radius, ulna) to the carpus.

The intrinsic ligaments of each carpal row (proximal and distal) consist of dorsal and palmar regions, connect two adjacent bones, and play a role in maintaining normal 


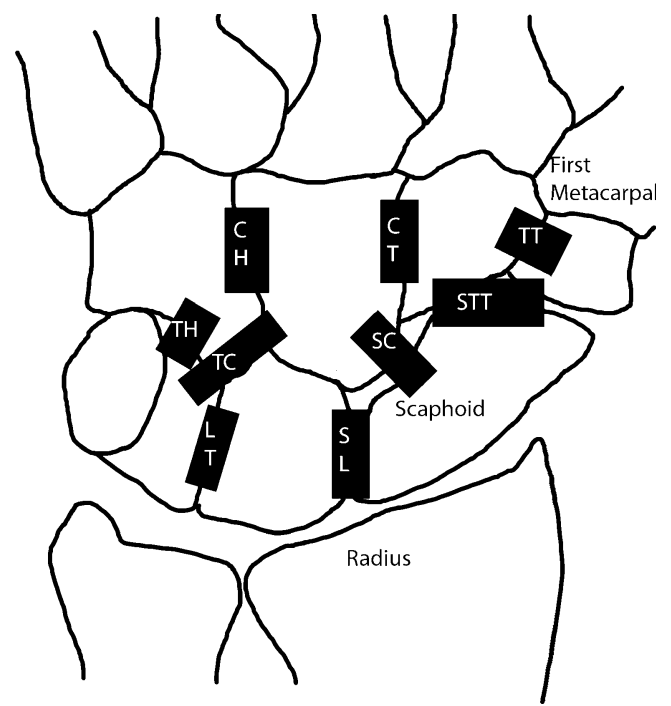

Fig. 1 Volar view of intrinsic ligaments $(C H$ capitohamate, $C T$ capitotrapezium, $T T$ trapeziotrapezoid, $T H$ triquetrohamate, $T C$ triquetrocapitate, SC scaphocapitate, STT scaphotrapeziotrapezoid, LT lunotriquetrum, $S L$ scapholunate)

biomechanics of the wrist [11]. The SL and LT ligaments are very important for maintaining carpal stability, connecting the proximal aspects of the scaphoid and lunate and the proximal portions of the lunate and triquetrum, respectively $[1,12]$. Both have dorsal, palmar and membranous portions $[1,13]$. With regard to those ligaments that connect the proximal and distal carpal rows, the arcuate ligament is composed of two arms, the TC ligament and the SC ligament. Their obliquely oriented fibers attach to the capitate, forming a supporting structure that links the proximal and distal carpal rows [1]. The TH ligament extends from the distal margin of the palmar cortex of the triquetrum, ulnar to the triquetrocapitate ligament, to the palmar cortex of the hamate $[1,2]$. The STT ligament connects the scaphoid to the trapezium and trapezoid, its fibers attaching to the radial and ulnar cortices of the distal pole of the scaphoid, forming a V-shaped structure. In general terms the STT and SC ligaments are a 'mirror image' of the $\mathrm{TH}$ and $\mathrm{TC}$ ligaments [1]. The intrinsic ligaments of the distal carpal row are the TT, CT and $\mathrm{CH}$ ligaments, connecting the trapezium to the trapezoid, the capitate to the trapezoid, and the capitate to the hamate, respectively. The last two ligaments also possess deep portions that are oriented differently from superficial portions [11].

Although there are many articles that describe the anatomy and magnetic resonance (MR) imaging characteristics of the SL and LT ligaments, the usefulness of different wrist positions in the assessment of these ligaments with MR imaging and MR arthrography, to the best of our knowledge, has not been investigated. The aim of this study was to demonstrate how radial and ulnar deviation of the wrist can influence the visualization of the intrinsic ligaments using MR imaging and MR arthrography in cadavers.

\section{Materials and methods}

Ten fresh human wrists, each cut across the forearm, were harvested from eight nonembalmed cadavers (six men and four women; age range at death 56-97 years; mean age 82.9 years) and used according to institutional guidelines. The cadaveric specimens were immediately stored at $-41^{\circ} \mathrm{C}$ in a freezer (Forma Bio-Freezer; Forma Scientific, Marietta, $\mathrm{OH}, \mathrm{USA}$ ) and were allowed to thaw at room temperature for $12 \mathrm{~h}$ prior to undergoing MR imaging. MR images were obtained with a $1.5 \mathrm{~T}$ scanner (Signa; GE Medical Systems, Milwaukee, WI, USA) with a standard eight-channel wrist array coil. The images were acquired before and after arthrography, in axial, sagittal and coronal planes, and in neutral position and radial and ulnar deviation. To obtain radial and ulnar deviation, we fastened the forearm in a plastic device, and the fingers were attached to each other and moved in the coronal plane until, based on resistance to further movement, the maximum degree of radial or ulnar deviation was achieved; at this point the fingers were fixed in the device. The following protocol was applied: precontrast proton density (PD)-weighted spin-echo [time to repeat (TR) 3,000 ms, time to echo (TE) $28 \mathrm{~ms}$, section thickness $2 \mathrm{~mm}$, no interslice spacing, number of signals acquired 2, field of view $7 \mathrm{~cm} \times 7 \mathrm{~cm}$, matrix 512 pixels $\times$ 256 pixels]; pre-contrast PD-weighted spin-echo with fat saturation (TR 3,000 ms, TE $28 \mathrm{~ms}$, section thickness $2 \mathrm{~mm}$, no interslice spacing, number of signals acquired 2 , field of view $7 \mathrm{~cm} \times 7 \mathrm{~cm}$, matrix 512 pixels $\times 256$ pixels); post-contrast $\mathrm{PD}$-weighted spin-echo (TR 3,000 ms, TE

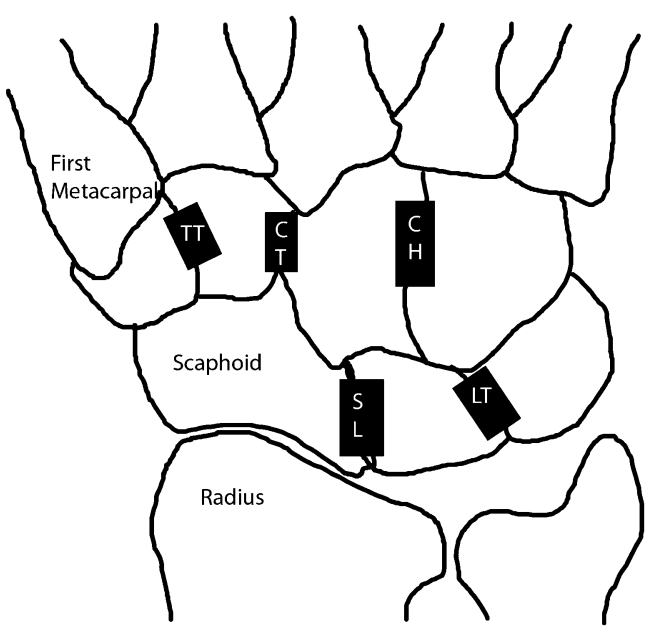

Fig. 2 Dorsal view of intrinsic ligaments (TT trapeziotrapezoid, $C T$ capitotrapezoid, $\mathrm{CH}$ capitohamate, $S L$ scapholunate, $L T$ lunotriquetrum) 
$28 \mathrm{~ms}$, section thickness $2 \mathrm{~mm}$, no interslice spacing, number of signals acquired 2, field of view $7 \mathrm{~cm} \times 7 \mathrm{~cm}$, matrix 512 pixels $\times 256$ pixels); and post-contrast $\mathrm{T} 1$ weighted spin-echo with fat saturation (TR $500 \mathrm{~ms}$, TE $22 \mathrm{~ms}$, section thickness $2 \mathrm{~mm}$, no interslice spacing, number of signals acquired 2, field of view $7 \mathrm{~cm} \times 7 \mathrm{~cm}$, matrix 512 pixels $\times 256$ pixels).

Two musculoskeletal radiologists (initials hidden) performed arthrography of the midcarpal, radiocarpal and distal radioulnar joints. A 22-gauge needle was used from a dorsal approach. A diluted gadopentetate solution was prepared with a mixture of $2 \mathrm{ml}$ of gadolinium dimeglumine (Magnevist; Bayer-Schering, Berlin, Germany) and $250 \mathrm{ml}$ of saline solution $(50 \%)$ and iodinated contrast agent (50\%) (Omnipaque 350; Bayer Healthcare Pharmaceuticals, Leverkusen, Germany). A total of $1.5 \mathrm{ml}$ was injected into the midcarpal joint, $1.5 \mathrm{ml}$ in the radiocarpal joint and $2 \mathrm{ml}$ in the distal radioulnar joint. When communicating defects were found, the injection was limited to one or two compartments, using a total of $5 \mathrm{ml}$.

After MR imaging had been completed, the cadaveric specimens were frozen again at $-41^{\circ} \mathrm{C}$ for more than $72 \mathrm{~h}$ and were subsequently cut in the axial, coronal, or sagittal plane with a band saw into $2 \mathrm{~mm}$-thick slices, so that anatomic slices, considered the standard reference, corresponded closely to the MR imaging planes (Fig. 3) The MRI plane that best demonstrated the ligament was used for sectioning purposes. The slices were cleaned with running water for macroscopic inspection. Each slice was photographed and radiographed.

We employed multiplanar slices and three different positions of the wrist (i.e., neutral, radial and ulnar deviation) to determine the best plane and position for visualization of each ligament. To accomplish this, two radiologists (initials hidden) analyzed the ten wrists in consensus, paying attention to the visibility of each ligament and grading them as visible or not visible. Both readers were involved in the anatomical study.

Two methods of measurement were used, in a position of maximum radial and ulnar deviation in each specimen, to obtain the wrist angulation and the width of the radiocarpal joint:

1. The wrist angulation was estimated in the coronal plane from images that showed the largest diameter of the capitate. In these images a line was drawn from the styloid process of the radius to the distal surface of the radius, close to the radioulnar joint, and then a second line in the long axis of the capitate was determined with DICOM software (Osirix 3.2.1; The Osirix Foundation, Geneva, Switzerland). The angle between these two lines was obtained and compared in radial and ulnar deviation (Fig. 4). The capitate was

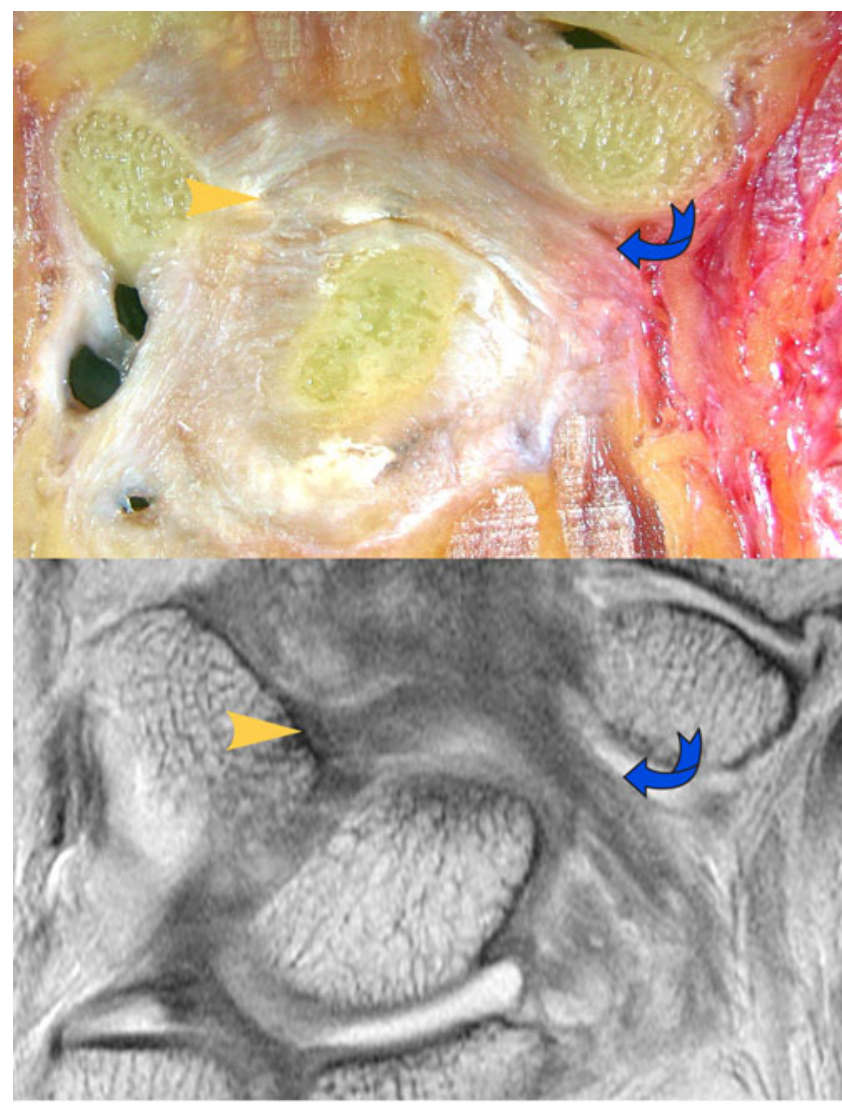

Fig. 3 Example of comparison between gross anatomy and MR imaging (Arrowhead triquetrocapitate, curved arrow scaphocapitate)

used as a reference because the trajectories of the hand during these movements, when they occur in a fixed plane, are circular, and the rotation in each plane takes place about a fixed axis that is located within the head of the capitate [14].

2. The width of the radiocarpal joint was calculated from the distance between the middle portion of the scaphoid and the radial cartilage in the radial side and across the LT ligament and the surface of the triangular fibrocartilage (TFC) in the ulnar side. We constructed all lines perpendicular to the surface of the scaphoid and LT ligament by using two lines, one to calculate the correct angulation and the other to measure the width in millimeters (Fig. 5). All measurements were made with the use of the Osirix DICOM viewer.

Student's $t$-test was performed with computer software (Microsoft Excel 2008; Microsoft, Redmond, WA, USA) to evaluate the measurements of the radiocarpal joint space. A $P$ value $<0.05$ was considered to indicate a significant difference. Three specimens with severe ligament tears that likely altered the biomechanics of the wrist were excluded from the statistical analysis. 
Fig. 4 Lines used in combination to obtain the carpal angle measurements, in radial (left) and ulnar (right) deviations

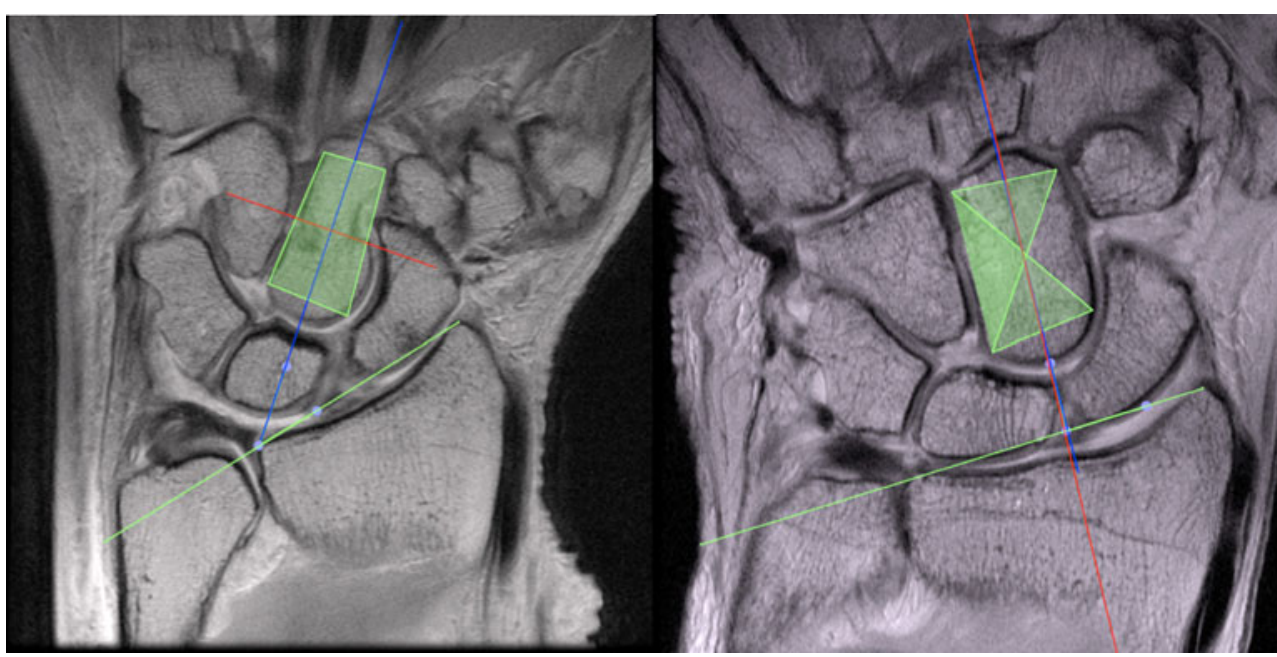

\section{Results}

The course and attachments of each intrinsic carpal ligament seen in the anatomic slices of all specimens corresponded closely to their appearance in the MR images. The differences in image analysis obtained before and after the intra-articular administration of contrast agent were immediately obvious upon analysis of the first two specimens, resulting in our decision to use the data derived from the MR arthrographic images alone for statistical purposes. Indeed, every single ligament was better depicted after MR arthrography, with the biggest differences in ease of visualization related to those ligaments located in the distal carpal row and those that crossed the midcarpal joint.

Radial and ulnar deviations changed the detectability of some ligaments. Radial deviation provided a better view of the ulnar side of the radiocarpal space and ulnar deviation accomplished the opposite (Fig. 6). With both of these maneuvers, portions of the midcarpal joint also widened to a small degree. The SL and LT ligaments were best depicted in the axial and coronal planes. The dorsal and palmar portions of the SL and LT ligaments were well seen with ulnar and radial deviation, as well as in the neutral position. The membranous portion of these ligaments was better visualized in the coronal plane, in all wrist positions (Fig. 7). The ligaments that cross the midcarpal joint were best depicted in the coronal plane. Radial and ulnar deviations led to little improvement in the visualization of the SC and TC ligaments (ulnar deviation) and the STT and TH ligaments (radial deviation). Concerning the ligaments of the distal carpal row, most of these were clearly defined both in the axial plane and, to a slightly lesser degree, in the coronal plane (neutral position) (Fig. 8).

The average angle of the capitate axis, as the wrist was moved from ulnar to radial deviation, was $38.4^{\circ}$ [standard deviation (SD) $7.38^{\circ}$ ]. The mean widths of the radiocarpal joint in the radial side were 0.62 (SD 0.14 ) $\mathrm{mm}, 1.55$ (SD $0.51) \mathrm{mm}$, and 2.0 (SD 0.31) $\mathrm{mm}$ and in the ulnar side 3.78

Fig. 5 Example of measurement of the width in the ulnar side, after radial deviation (left image) and in the radial side, after ulnar deviation (right image). Each image depicts two lines, one for the angle (left side) and the other for the width (right side)
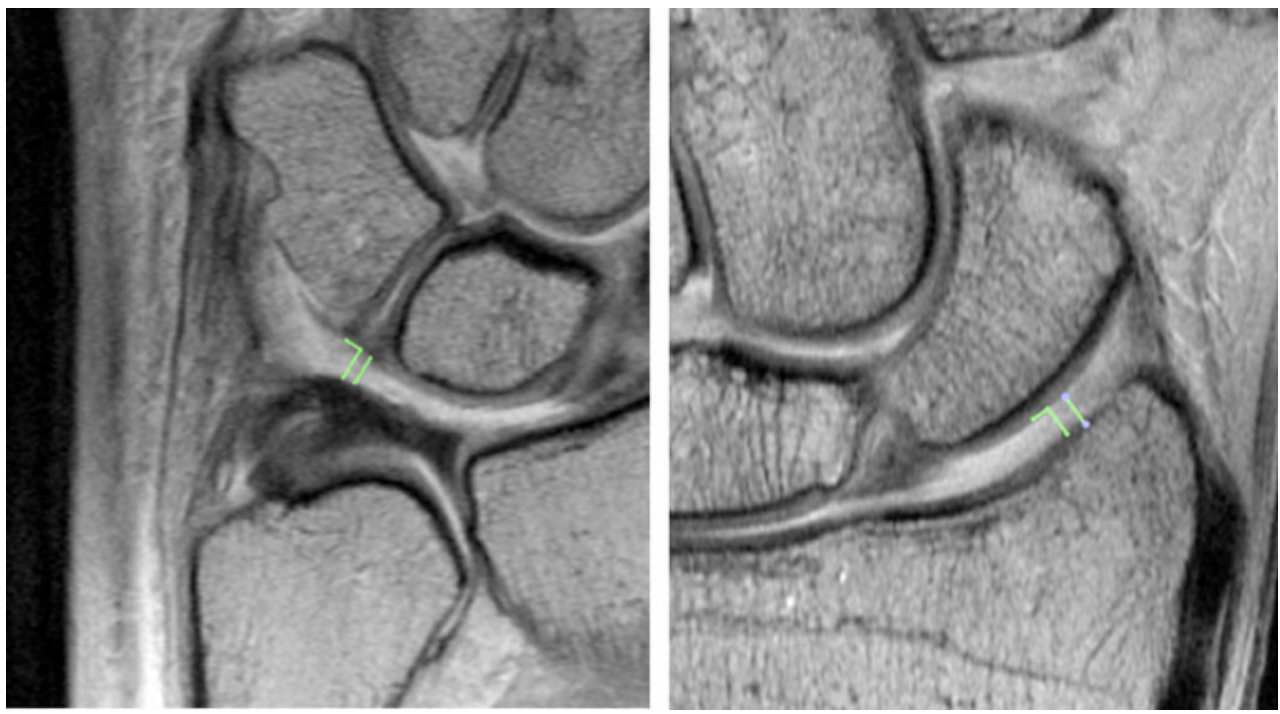
Fig. 6 MR arthrograms in the coronal plane in radial deviation (left image) better depicting the LT ligament (arrow) and ulnar deviation (right image) better showing the SL ligament (arrowhead)
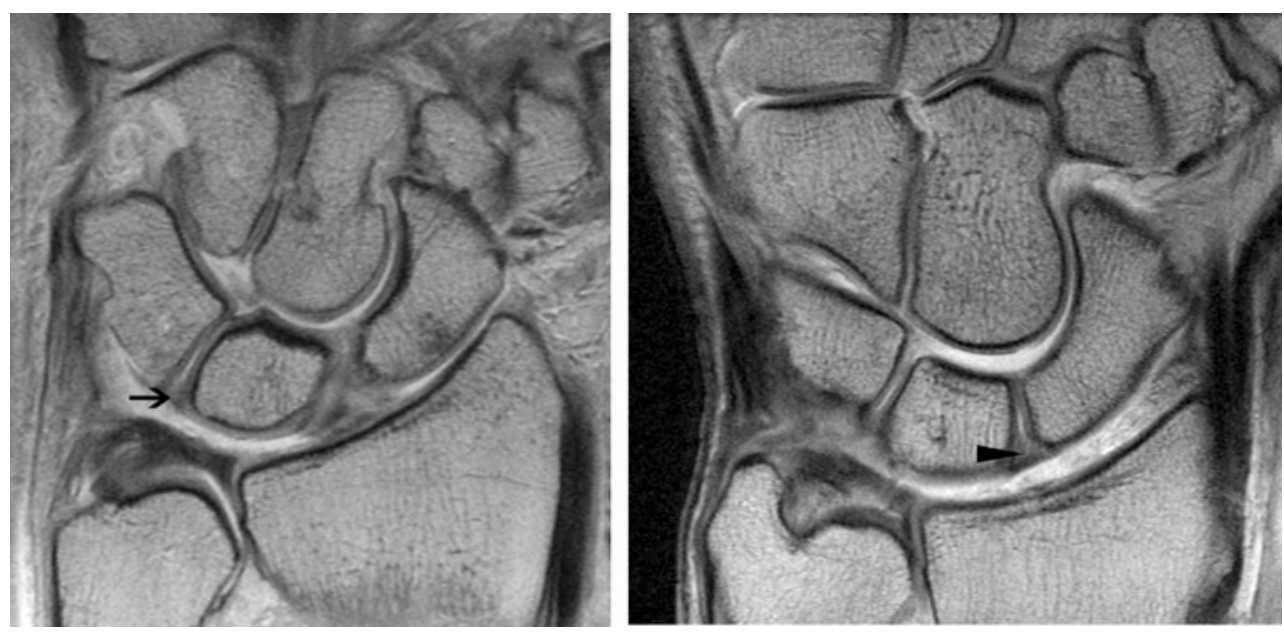

Fig. 7 Detectability of different regions of the scapholunate and lunotriquetral ligaments by MR arthrography in radial deviation (top), ulnar deviation (middle) and neutral position (bottom). $Y$-axis number of ligaments observed, $X$-axis portions of the ligaments. DSL dorsal scapholunate, $M S L$ membranous scapholunate, $P S L$ palmar scapholunate, $D L T$ dorsal lunotriquetral, $M L T$ membranous lunotriquetral, PLT palmar lunotriquetral
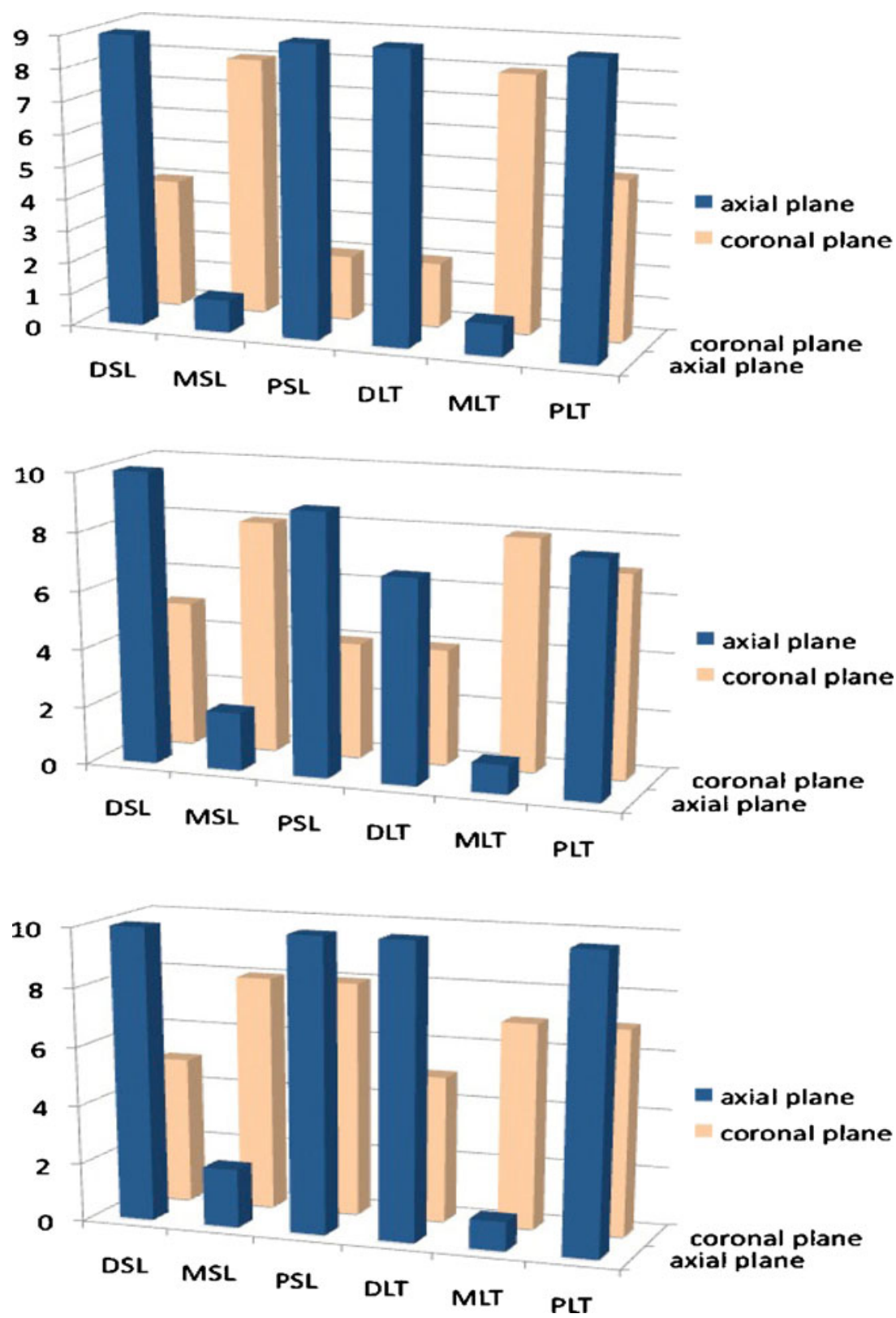
Fig. 8 Detectability of the scaphotrapezotrapezoid (STT), scaphocapitate $(S C)$, triquetrocapitate $(T C)$, triquetrohamate $(T H)$, trapeziotrapezoid (TT), capitotrapezoid $(C T)$ and capitohamate $(\mathrm{CH})$ in neutral $(N)$ position and in radial $(R D)$ and ulnar ( $U D$ deviation). $Y$-axis number of ligaments observed, $X$-axis planes and positions of study

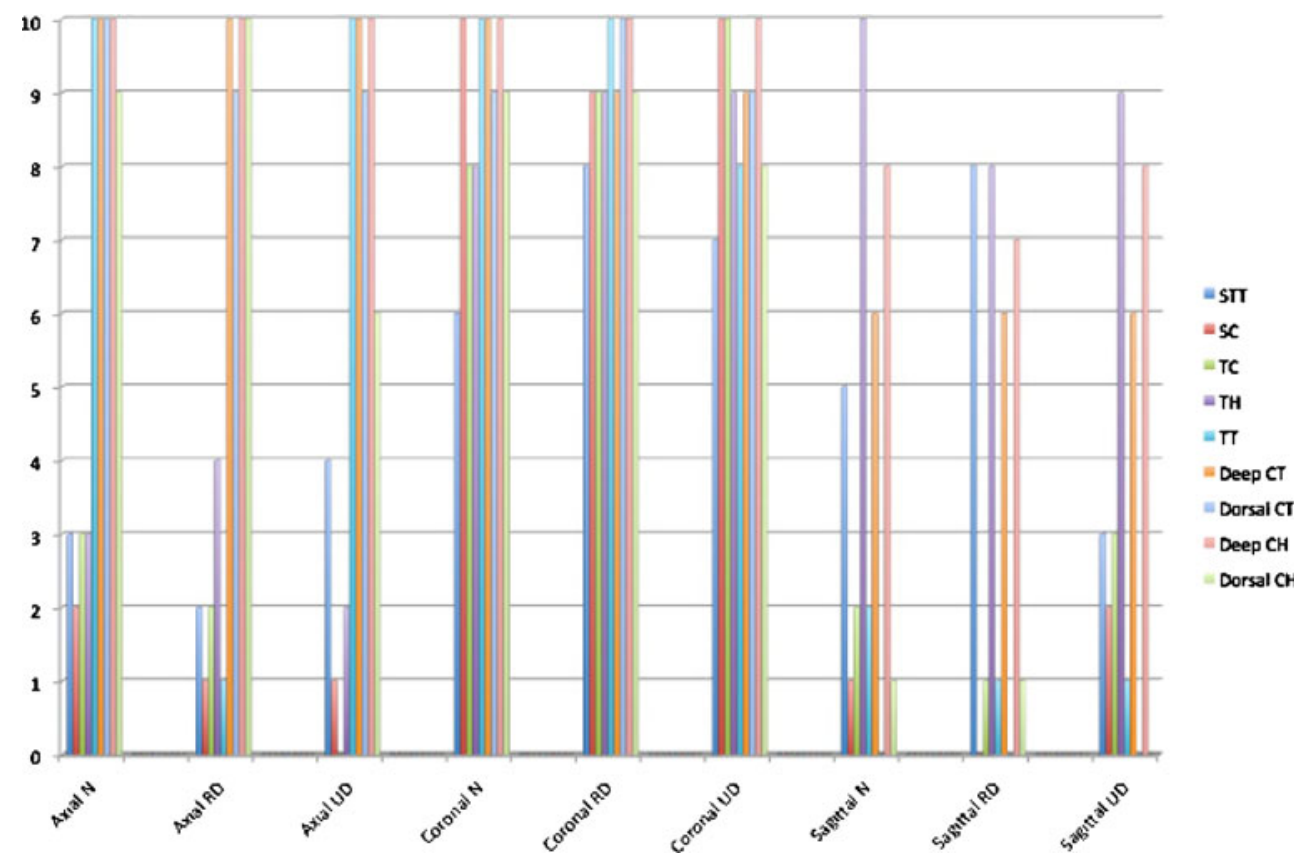

(SD 1.33) mm, 2.25 (SD 1.17) $\mathrm{mm}$ and $1.16(\mathrm{SD} \mathrm{0.41)} \mathrm{mm}$ in radial deviation, neutral position, and ulnar deviation of the wrist, respectively (Table 1).

Significant differences were noted in the widths of the radial and ulnar sides of the radiocarpal joint during radial and ulnar deviations. Radial deviation opened the ulnar side of the radiocarpal joint approximately $1.53 \mathrm{~mm}(P=0.0005)$ and diminished the radial side about $0.93 \mathrm{~mm}(P=0.0002)$, and ulnar deviation opened the radial side approximately $0.45 \mathrm{~mm}(P=0.007)$ and diminished the ulnar side by about $1.09 \mathrm{~mm}(P=0.004)$.

\section{Discussion}

Radiographs obtained with radial and ulnar deviation are reported to improve the evaluation of major intrinsic ligaments in clinical practice, since the most common carpal instability is secondary to ligamentous disruption between the scaphoid and lunate, and between the lunate and triquetrum [15].

According to Manton et al., conventional MR images in cases of partial tears of the SL and LT ligaments appear to be of limited accuracy [13]. For this reason, the injection of intra-articular contrast media has been justified [10]. MR arthrography combines the advantage of conventional MR imaging with that of arthrography by improving the visualization of small structures, such as the intrinsic ligaments of the wrist $[9,10,16]$. Tricompartmental arthrographic injection of the wrist, as suggested by Robinson et al., has been reported to allow best evaluation of the intrinsic ligaments [17].

In our investigation, in order to exaggerate the changes in the dimensions of the radiocarpal space during radial and

Table 1 Measurements of the wrist aperture in millimeters, and angles in degrees ( $R S$ radial side, US ulnar side, dev deviation)

\begin{tabular}{|c|c|c|c|c|c|c|c|}
\hline Specimen & RS neutral & US neutral & RS radial dev & US radial dev & RS ulnar dev & US ulnar dev & Angle \\
\hline 1 & 1.79 & 1.21 & 0.73 & 3.67 & 2.2 & 0.75 & 41.6 \\
\hline 2 & 2.01 & 3.20 & 0.48 & 5.31 & 1.68 & 1.44 & 35.7 \\
\hline 3 & 2.37 & 1.88 & 0.76 & 2.67 & 2.13 & 1.01 & 34.4 \\
\hline 4 & 1.46 & 1.08 & 0.34 & 1.69 & 0.91 & 0.82 & 55.2 \\
\hline 5 & 0.96 & 1.83 & 0.53 & 4.49 & 2.02 & 1.51 & 35 \\
\hline 6 & 1.83 & 1.58 & 0.62 & 3.75 & 2.2 & 1.06 & 32.8 \\
\hline 7 & 0.98 & 2.86 & 0.57 & 5.37 & 1.47 & 0.79 & 36.6 \\
\hline 8 & 1.95 & 4.97 & 0.80 & 5.29 & 2.22 & 1.89 & 46 \\
\hline 9 & 0.90 & 2.31 & 0.70 & 3.24 & 2.27 & 1.58 & 30 \\
\hline 10 & 1.23 & 1.57 & 0.65 & 2.27 & 1.66 & 0.73 & 37.4 \\
\hline
\end{tabular}


ulnar deviation, we applied maximum radial and ulnar deviation to the wrists. In addition, the angulation of the capitate was measured using principles similar to those that were developed for quantitative measurements of radiographs, such as the amount of translation of the carpus in pathological conditions (e.g., carpal collapse) [14, 18]. The average measured angle was below the range of motion that has been reported in a healthy population, $53^{\circ}$ according to Youm et al. [14] We attribute this discrepancy to degenerative diseases and intrinsic stiffness when dealing with cadaveric wrist specimens derived from elderly donors. Some measurements of the width of the radiocarpal joint during ulnar deviation, as shown in Table 1, as well as differences in the detectability of the ligaments (i.e., some showed an improvement while others did not) can be attributed to the same factors. The lack of any data related to the width of the radiocarpal joint in asymptomatic volunteers during radial and ulnar deviation and the small sample of cadaveric specimens were other limitations of our study.

While other factors such as ligament tension clearly may contribute to changes in the visibility of some of these ligaments, we believe that the dominant factors relate to the width of the radiocarpal joint and the changes in this width that accompany modifications in wrist position, as well as the articular distension provided by MR arthrography[19. An improvement in the analysis of the intrinsic ligaments of the wrist may be possible through the judicious use of radial and ulnar deviation, especially when combined with MR arthrography.

Open Access This article is distributed under the terms of the Creative Commons Attribution Noncommercial License which permits any noncommercial use, distribution, and reproduction in any medium, provided the original author(s) and source are credited.

\section{References}

1. Berger RA. The anatomy of the ligaments of the wrist and distal radioulnar joints. Clin Orthop Relat Res. 2001;(383):32-40

2. Brown RR, Fliszar E, Cotten A, Trudell D, Resnick D. Extrinsic and intrinsic ligaments of the wrist: normal and pathologic anatomy at MR arthrography with three-compartment enhancement. Radiographics. 1998;18:667-74.

3. Chang W, Peduto AJ, Aguiar RO, Trudell DJ, Resnick DL. Arcuate ligament of the wrist: normal MR appearance and its relationship to palmar midcarpal instability: a cadaveric study. Skeletal Radiol. 2007;36:641-5.

4. Manaster BJ. Digital wrist arthrography: precision in determining the site of radiocarpal-midcarpal communication. AJR Am J Roentgenol. 1986;147:563-6.

5. Mayfield JK, Johnson RP, Kilcoyne RF. The ligaments of the human wrist and their functional significance. Anat Rec. 1976;186:417-28.

6. Rominger MB, Bernreuter WK, Kenney PJ, Lee DH. MR imaging of anatomy and tears of wrist ligaments. Radiographics. 1993;13:1233-46; discussion 1247-8.

7. Taleisnik J. The ligaments of the wrist. J Hand Surg [Am]. 1976;1: $110-8$.

8. Theumann N, Favarger N, Schnyder P, Meuli R. Wrist ligament injuries: value of post-arthrography computed tomography. Skeletal Radiol. 2001;30:88-93.

9. Montet X, Eberlin JL, Bianchi S, Fasel J. Assessment of intraarticular volume of the wrist: a comparative study between CTarthrography and dissection. Surg Radiol Anat. 2005;27:444-9.

10. Cerezal L, Abascal F, Garcia-Valtuille R, Del Pinal F. Wrist MR arthrography: how, why, when. Radiol Clin North Am. 2005;43:70931.

11. An K-N, Berger RA, Cooney WP. Biomechanics of the wrist joint. New York: Springer; 1991.

12. Timins ME, Jahnke JP, Krah SF, Erickson SJ, Carrera GF. MR imaging of the major carpal stabilizing ligaments: normal anatomy and clinical examples. Radiographics. 1995;15:575-87.

13. Manton GL, Schweitzer ME, Weishaupt D, Morrison WB, Osterman AL, Culp RW, et al. Partial interosseous ligament tears of the wrist: difficulty in utilizing either primary or secondary MRI signs. J Comput Assist Tomogr. 2001;25:671-6.

14. Youm Y, McMurtry RY, Flatt AE, Gillespie TE. Kinematics of the wrist. I. An experimental study of radial-ulnar deviation and flexion-extension. J Bone Joint Surg Am. 1978;60:423-31.

15. Taleisnik J. Current concepts review. Carpal instability. J Bone Joint Surg Am. 1988;70:1262-8.

16. Berna-Serna JD, Martinez F, Reus M, Alonso J, Domenech-Ratto G. Wrist arthrography: a simple method. Eur Radiol. 2006;16:46972.

17. Robinson G, Chung T, Finlay K, Friedman L. Axial oblique MR imaging of the intrinsic ligaments of the wrist: initial experience. Skeletal Radiol. 2006;35:765-73.

18. McMurtry RY, Youm Y, Flatt AE, Gillespie TE. Kinematics of the wrist. II. Clinical applications. J Bone Joint Surg Am. 1978;60: 955-61.

19. Ton ER, Pattynama PM, Bloem JL, Obermann WR. Interosseous ligaments: device for applying stress in wrist MR imaging. Radiology. 1995;196:863-4. 